\title{
Reliability Evaluation of Low-voltage Switchgear Based on Maximum Entropy Principle
}

\author{
Zhang Zhigang ${ }^{1}$, Wang Jingqin ${ }^{2}$, Wang $\mathrm{Li}^{3}$, Wang Meng ${ }^{4}$ \\ 1,2,3,4 Province-Ministry Joint Key Laboratory of Electromagnetic Field and Electrical Apparatus \\ Reliability, Hebei University of Technology, Tianjin, China \\ ${ }^{2}$ Changshu Switchgear Manufactory Co. Ltd., Changshu, China \\ Correspondong author, e-mail: zhangzhigang@cs-kg.com ${ }^{1,}$ jqwang@hebut.edu.cn² \\ Iwangeducation@163.com³ ; 465276457@qq.com ${ }^{4}$
}

\begin{abstract}
In this paper, based on the definition of two-parameter joint entropy and the maximum entropy principle, a method was proposed to determine the prior distribution by using the maximum entropy method in the reliability evaluation of low-voltage switchgear. The maximum entropy method takes kinds of priori information as different constraints. The optimal prior distribution was selected by maximizing entropy under these constraints, which not only contains the known prior information but also tries to avoid the introduction of other assumption information. Based on non-parametric bootstrap method, the hyperparameters of prior distribution is obtained by two-order moment of prior information. Finally, with the bootstrap method, the prior distribution robustness and the posterior robustness were analyzed, and the posterior mean time between failures for the low-voltage switchgear was estimated.
\end{abstract}

Keywords: prior distribution, maximum entropy, low-voltage switchgear, reliability evaluation

Copyright @ 2017 Universitas Ahmad Dahlan. All rights reserved.

\section{Introduction}

Low-voltage switchgear is responsible for power control, protection, measurement, transformation and distribution in low-voltage power supply system. For the reliability evaluation of low-voltage switchgear with high reliability and long life, traditional reliability assessment method needs to obtain sufficient data by a large number of sample life tests which is timeconsuming, costly and inefficient [1]. Therefore, the rational use of empirical and historical data to determine priori distribution can lay a solid theoretical foundation for the reliability evaluation of low-voltage switchgear.

Bayes method can make good use of not only the field test information, but also priori information, such as historical test information, test information for similar models and the same type products with different conditions, and so on. And the priori information can be used to get the priori distribution which increases the failure data. This method has been applied in most fields, such as medical system [2], web [3], electrical engineering [4], finance [5], and speech recognition [6]. While, there is no discussion about low-voltage switchgear.

In this paper, Bayes method is used to evaluate the reliability of low-voltage switchgear. The priori distribution of low-voltage switchgear is determined by maximum entropy method which avoids the introduction of other assumption information because of the using of priori information. According to maximum entropy principle, priori information can be taken as different contrains, and the optimal prior distribution can be selected by maximizing entropy under these constraints. The non-parametric bootstrap method is used to expand data capacity and then hyper-parameters of priori distribution is eatimated. Finally, with the bootstrap method, the prior distribution robustness and the posterior robustness is analyzed, and the posterior mean time between failures for the low-voltage switchgear is estimated.

\section{Maximum Entropy Method}

During the practical application of Bayes theory, there is some available prior information, but you would like try not to introduce any other assumptive information except the priori information. This problem can be effectively solved by the concept of entropy [7]. Entropy 
is definited that when $\Theta$ is discrete, $\pi$ is the probability density of $\Theta, \varepsilon_{N}(\pi)$ means the entropy of $\pi$. And $\varepsilon_{N}(\pi)$ is defined as:

$$
\varepsilon_{N}(\pi)=-\sum_{\Theta} \pi\left(\theta_{i}\right) \log \pi\left(\theta_{i}\right)
$$

If $\pi\left(\theta_{i}\right)=0, \pi\left(\theta_{i}\right) \log \pi\left(\theta_{i}\right)$ can be defined as zero.

It is assumed that part of prior information about $\theta$ has been known, then these information can be expressed by some constraints of $\pi\left(\theta_{i}\right)$. The assumption is:

$$
E^{\pi}\left[g_{k}(\theta)\right]=\sum_{i} \pi\left(\theta_{i}\right) g_{k}\left(\theta_{i}\right)=\mu_{k}(k=1, \ldots, m)
$$
solution is:

In the premise of meeting the above constraints (including $\Sigma \pi\left(\theta_{i}\right)=1$ ), the obtained

$$
\bar{\pi}\left(\theta_{i}\right)=\frac{\exp \left\{\sum_{k=1}^{m} \lambda_{k} g_{k}\left(\theta_{i}\right)\right\}}{\sum_{i} \exp \left\{\sum_{k=1}^{m} \lambda_{k} g_{k}\left(\theta_{i}\right)\right\}}
$$

Where, $\lambda_{k}$ is the constant determined by the above-mentioned constraints.

When $\Theta$ is continuous, Jaynes defined entropy as [8,9]:

$$
\varepsilon_{N}(\pi)=-E^{\pi}\left[\log \frac{\pi(\theta)}{\pi_{0}(\theta)}\right]=-\int \pi(\theta) \log \left(\frac{\pi(\theta)}{\pi_{0}(\theta)}\right) d \theta
$$

Where, $\Pi_{0}(\theta)$ is the non-information prior. While people can still use the following definition.

Part of prior information are expressed as follows:

$$
E^{\pi}\left[g_{k}(\theta)\right]=\int_{\Theta} g_{k}(\theta) \pi(\theta) d \theta=\mu_{k}(k=1, \ldots, m)
$$

The prior density that $\varepsilon_{N}(\pi)$ can be got the maximum is determined by formula (6).

$$
\bar{\pi}(\theta)=\frac{\pi_{0}(\theta) \exp \left[\sum_{k=1}^{m} \lambda_{k} g_{k}(\theta)\right]}{\int_{\Theta} \pi_{0}(\theta) \exp \left[\sum_{k=1}^{m} \lambda_{k} g_{k}(\theta)\right] \mathrm{d} \theta}
$$

Where, $\lambda_{k}$ is a constant determined by formula (7).

$$
\varepsilon_{N}(\pi)=-E^{\pi}\left[\log \frac{\pi(\theta)}{\pi_{0}(\theta)}\right]=-\int \pi(\theta) \log \left(\frac{\pi(\theta)}{\pi_{0}(\theta)}\right) d \theta
$$

\section{The Maximum Entropy Method for the Prior Distribution of Low-voltage Switchgear}

\subsection{The Joint Prior Distribution Determined by the Maximum Entropy}

The basic viewpoint of maximum entropy principle is that, to accurately infer the system state in the case where there is only part of the information, a reasonable state should be selected to meet the constraint condition and maximize the entropy.

Formula (8) is the expression of the joint prior distribution $\pi(\eta, m)$, and formula (9) to formula (11) are its constraint conditions.

$$
\max H(\eta, m)=-\int_{-\infty}^{+\infty} \int_{-\infty}^{+\infty} \pi(\eta, m) \ln \pi(\eta, m) d \eta d m
$$




$$
\begin{aligned}
& \int_{-\infty}^{+\infty} \int_{-\infty}^{+\infty} \pi(\eta, m) d \eta d m=1 \\
& \int_{-\infty}^{+\infty} \int_{-\infty}^{+\infty} \eta^{i} \pi(\eta, m) d \eta d m=E\left(\eta^{i}\right)=\eta_{i}, i=1, \ldots k \\
& \int_{-\infty}^{+\infty} \int_{-\infty}^{+\infty} m^{i} \pi(\eta, m) d \eta d m=E\left(m^{j}\right)=m_{j}, j=1, \ldots n
\end{aligned}
$$

Where, $\eta_{i}, m_{j}$ are the origin moments of $i$ order and $j$ order of two-parameter $\eta$ and $m$ respectively; $k$ and $n$ is the highest order of two-parameter origin moment respectively.

If one-order moment and two-order moment are exist, when $k=n=2$, by constructing and solving the auxiliary fonctionelle of joint density function, $\Pi(\eta, m)$ can be known $[10,11]$,

$$
\pi(\eta, m)=\frac{1}{2 \pi \sigma_{\eta} \sigma_{m}} \exp \left(-\frac{(\eta-\bar{\eta})^{2}}{2 \sigma_{\eta}^{2}}-\frac{(m-\bar{m})^{2}}{2 \sigma_{m}^{2}}\right)
$$

\subsection{Parameter Determination of Prior Distribution}

The bivariate normal prior distribution contains hyper-parameters which can be calculated by prior information. So, for the problems of small samples with insufficient historical data, the small sample data can be re-sampled to expand the data capacity by using bootstrap method to simulate the general characteristics and determine the prior information $[12,13]$.

The methods of determining hyper-parameter by using non-parametric bootstrap method are described as follows [14].

$T=\left(t_{1}, t_{2}, \ldots t_{n}\right)$ denotes a set of obtained samples with total failure. The distribution parameter samples can be obtained by the following steps.

1) The self-help sample $T^{*}=\left(t_{1}^{*}, t_{2}^{*}, \ldots, t_{\mathrm{n}}^{*}\right)$ can be obtained by carrying out sampling with replacement for $T=\left(t_{1}, t_{2}, \ldots t_{n}\right)$;

2) $\hat{m}$ and $\hat{\eta}$ can be got from the maximum likelihood estimation with the self-help sample $T^{*}=\left(t_{1}^{*}, t_{2}^{*}, \ldots, t_{\mathrm{n}}^{*}\right)$; be got:

3) Repeat the previous two steps for $N$ times, then the estimated parametric sample can

$$
\left\{\left(\hat{m}_{1}, \hat{\eta}_{1}\right),\left(\hat{m}_{2}, \hat{\eta}_{2}\right), \ldots,\left(\hat{m}_{N}, \hat{\eta}_{N}\right)\right\}
$$

4) The expectations and variances of $m$ and $\eta$ can be got by the obtained estimation parametric samples.

$\overline{\hat{m}}$ and $S_{\mathrm{m}}^{2}$ denote the expectation and variance of the shape parameter $m$ respectively, $\overline{\hat{\eta}}$ and $S_{\eta}^{2}$ denote the expectation and variance of the scale parameter $\eta$. Then:

$$
\begin{aligned}
& \overline{\hat{m}}=\frac{1}{n} \sum_{\mathrm{j}=1}^{n} \hat{m}_{j}, S_{m}^{2}=\frac{1}{n-1} \sum_{\mathrm{j}=1}^{n}\left(\hat{m}_{j}-\overline{\hat{m}}\right)^{2} \\
& \overline{\hat{\eta}}=\frac{1}{k} \sum_{\mathrm{i}=1}^{k} \hat{\eta}_{i}, S_{\eta}^{2}=\frac{1}{k-1} \sum_{\mathrm{i}=1}^{k}\left(\hat{\eta}_{i}-\overline{\hat{\eta}}\right)^{2}
\end{aligned}
$$

By calculating the transcendental moment, the hyper-parameter expressions of the prior distribution can be obtained:

$$
\bar{m}=\overline{\hat{m}}, \sigma_{\mathrm{m}}^{2}=S_{\mathrm{m}}^{2}, \bar{\eta}=\overline{\hat{\eta}}, \sigma_{\eta}^{2}=S_{\eta}^{2}
$$


The life distribution of a certain low-voltage switchgear obeys Weibull $(3600,1.5) .30$ samples are randomly generated as the historical failure data, that is the prior information, as shown in Table 1.

Table 1. Historical Failure Data of Low-voltage Switchgear (unit: hours)

$\begin{array}{ccccccccc}6439 & 3432 & 2201 & 1273 & 809 & 5552 & 3746 & 5365 & 5140 \\ 5328 & 280 & 3315 & 1849 & 6039 & 4144 & 3090 & 2384 & 2050 \\ 7962 & 3044 & 3254 & 1950 & 2400 & 2034 & 1330 & 1607 & 840\end{array}$

The parameters of the bivariate normal prior distribution are as follows:

$$
\bar{m}=1.69, \sigma_{\mathrm{m}}^{2}=0.0509, \bar{\eta}=3740, \sigma_{\eta}^{2}=180121
$$

Then the prior density function of low-voltage switchgear can be got:

$$
\pi(\eta, m)=\frac{1}{18336.3178 \pi} \exp \left(-\frac{(\eta-3740)^{2}}{360242}-\frac{(m-\bar{m})^{2}}{0.1018}\right)
$$

\subsection{Robust Analysis of Prior Distribution}

Before using the prior distribution, it is necessary to analyze its impact on the posteriori statistical inference results, which is known as the robust analysis of prior distributions [15, 16].

A way to deal with priori uncertainty is to try to exclude the priori which should not be considered in the aspect of data from the considered priori. Marginal density $m^{*}(t / \pi)$ can play a very important role because it is known as the relative likelihood marginal distribution functions. The marginal distribution values of relative likelihood functions represent the relative probability of field test samples with $\pi(\eta, m)$ as the prior distribution.

$m^{*}\left(t_{i} / \pi\right), i=1, \ldots N$ can be calculated when the field samples $\left(t_{1}, t_{2}, \ldots t_{N}\right)$ are obtained. The prior distribution can be considered conforming to the field test data, which shows the prior distribution $\pi(\eta, m)$ can be determined to be robust, if the value of $m^{*}\left(t_{i} / \pi\right)$ is not particularly small. For two-parameter Weibull distribution, the relative marginal likelihood function of joint prior distribution $\pi(\eta, m)$ is [17]:

$$
m^{*}(t / \pi)=\frac{m(t / \pi)}{\sup _{i} m(t / \pi)}=\frac{\int_{-\infty}^{+\infty} \int_{-\infty}^{+\infty} f(t / \eta, m) \pi(\eta, m) d \eta d m}{\max _{t} \int_{-\infty}^{+\infty} \int_{-\infty}^{+\infty} f(t / \eta, m) \pi(\eta, m) d \eta d m}
$$

To ensure the compatibility of prior information and the field testing information, the following test data $\left(T_{1}, \ldots T_{10}\right)=(885,2165,3560,9001,2036,1110,5602,1630,7187,3720)$ as the field test information will be put into the following formula,

$$
\mathrm{m}(T \mid \pi)=\left(\frac{m}{\eta}\right)^{N} \prod_{i=1}^{N}\left(\frac{t_{i}}{\eta}\right)^{m-1} \exp \left(-\sum_{i=1}^{N}\left(\frac{t_{i}}{\eta}\right)^{m}\right) \frac{1}{2 \pi \sigma_{n} \sigma_{m}} \exp \left[-\frac{\left(\eta-\mu_{\alpha}\right)^{2}}{2 \sigma_{n}^{2}}-\frac{\left(m-\mu_{m}\right)^{2}}{2 \sigma_{m}^{2}}\right] d \eta d m
$$

The relative marginal likelihood function values can be summarized in Table 2.

Table 2. Relative Marginal Likelihood Function Values of Prior Distribution

\begin{tabular}{ccccc}
\hline The first group & The second group & The third group & The forth group & The fifth group \\
\hline 0.5518 & 7.295 & 3.536 & 48.81 & 0.1241 \\
The sixth group & The seventh group & The eighth group & The ninth group & The tenth group \\
3.719 & 5.701 & 2.568 & 0.5819 & 91.73 \\
\hline
\end{tabular}




\section{The Posterior Distribution and Reliability Estimation of Low-voltage Switchgear}

Based on Bayes principle, it is assumed that the field life data of low-voltage switchgear is $T=\left(t_{1}, t_{2}, \ldots t_{n}\right)$, then the likelihood function can be expressed as:

$$
p\left(t_{1}, t_{2}, t_{3} \ldots t_{n} \mid \eta, m\right)=\left(\frac{m}{\eta}\right)^{n} \prod_{i=1}^{n}\left(\frac{t_{i}}{\eta}\right)^{m-1} \exp \left(-\sum_{i=1}^{n}\left(\frac{t_{i}}{\eta}\right)^{m}\right)
$$

The joint posterior distribution of parameter $\eta$ and $m$ can be expressed as:

$$
h\left(\eta, m \mid t_{1}, t_{2} \cdots t_{\mathrm{n}}\right)=\frac{p\left(t_{1}, t_{2} \cdots t_{\mathrm{n}} \mid \eta, m\right) \pi(\eta, m)}{\int_{-\infty}^{+\infty} \int_{-\infty}^{+\infty} p\left(t_{1}, t_{2} \cdots t_{n} \mid \eta, m\right) \pi(\eta, m) d \eta d m}
$$

Then the point estimate of the posterior distribution can be obtained:

$$
\hat{\eta}=E\left(\eta \mid t_{1}, t_{2} \ldots t_{n}\right)=3960, \hat{m}=E\left(m \mid t_{1}, \mathrm{t}_{2} \ldots t_{n}\right)=1.5466
$$
switchgear is:

Finally, the estimation of posterior mean time between failure of the low-voltage

$$
\overline{M T B F}=\hat{\eta} \Gamma(1+1 / \hat{m})=3562 \mathrm{~h}
$$

\section{The Posterior Robustness Analysis of Low-voltage Switchgear}

Formula (12) can be rewrittened as follows:

$$
\Gamma: \pi(\eta, \mathrm{m}) \sim N\left(\mu_{\eta}, \mu_{m}, \sigma_{\eta}^{2}, \sigma_{m}^{2}\right)
$$

Where,

$$
\underline{\mu}_{\eta} \leq \mu_{\eta} \leq \bar{\mu}_{\eta}, \underline{\mu}_{m} \leq \mu_{m} \leq \bar{\mu}_{m}, \underline{\sigma_{\eta}^{2}} \leq \sigma_{\eta}^{2} \leq \overline{\sigma_{\eta}^{2}}, \underline{\sigma_{m}^{2}} \leq \sigma_{m}^{2} \leq \overline{\sigma_{m}^{2}}
$$

$C_{\eta}$ and $C_{m}$ are the value intervals of $\eta$ and $m$, which is determined by the experience of experts. Also it is assumed that the acceptable maximum posterior expected loss within this confidence interval is $\gamma$. The posterior distribution $\pi(\eta, m / T)$ can be obtained according to the Bayes formula after the field date $T$ is obtained. The parametric range of prior distribution whose posterior expected loss is no more than $y$ can be calculated by:

$$
1-\int_{\eta \in C_{\eta}} \int_{m \in C_{m}} \pi(\eta, m \mid T) d \eta d m \leq \gamma
$$

In fact, it is difficult to calculate the parametric range of prior distribution by sloving the inequality (27). However, prior information can be used to get a set of parameters, based on which it is assumed that some of these parameters are constant, then change other parameters to get the approximate range of parameters.

In addition, by combining with the prior information and self-help sample methods, the sample mean and sample variance of the parametric samples can be obtained:

$$
\overline{\hat{m}}=1.44, \quad \mathrm{~S}_{m}^{2}=0.025, \quad \overline{\hat{\eta}}=2962, \quad \mathrm{~S}_{\eta}^{2}=1.98 \times 10^{5}
$$

Then a set of hyper-parameters of prior distribution can be got:

$$
\mu_{m}=1.44, \mu_{\eta}=2962, \sigma_{m}^{2}=0.025, \sigma_{\eta}^{2}=1.98 \times 10^{5}
$$


A set of field samples $T$ can be obtained by sampling method, as shown in Table 3 .

Table 3. A set of Field Samples With Censored Data (unit: hours)

\begin{tabular}{cccccccc}
\hline Complete failure data & 203 & 323 & 934 & 1583 & 1947 & 1961 & 3206 \\
\hline Censored data & 783 & 1353 & 1016 & 3097 & 3300 & & \\
\hline
\end{tabular}

The posterior distribution $\pi(\eta, m / T)$ can be obtained based on the test information of field samples.

To judge the robustness of the posterior distribution, it is necessary to know the parameter range of the prior distribution. The most commonly used method to get the range is by solving marginal distribution of every parametric obtained based on the posterior distribution. When solving, it is adopted to fix three parameters and chang another parameter. Then the parametric range of prior distribution is got by analyzing the obtained posterior expected loss.

According to their experience, experts provide the confidence intervals $C_{m}=(1,1.7)$, $C_{n}=(2500,3500)$ and the acceptable maximum posterior expected loss $y=0.2$. Other parameters in the prior distribution are fixed, and $\mu_{\eta}$ is calculated through formula (30) with 50 as the unit and the value got from 2500 to 3500 in turn.

$$
\int_{\eta \in C_{\eta}}\left[\int_{0}^{+\infty} \pi(\eta, m \mid T) d m\right] d \eta
$$

Where, $\mu_{m}=1.44, \sigma_{m}^{2}=0.025, \sigma_{\eta}^{2}=1.98 \times 10^{5}$. Results are summarized in Figure.1.

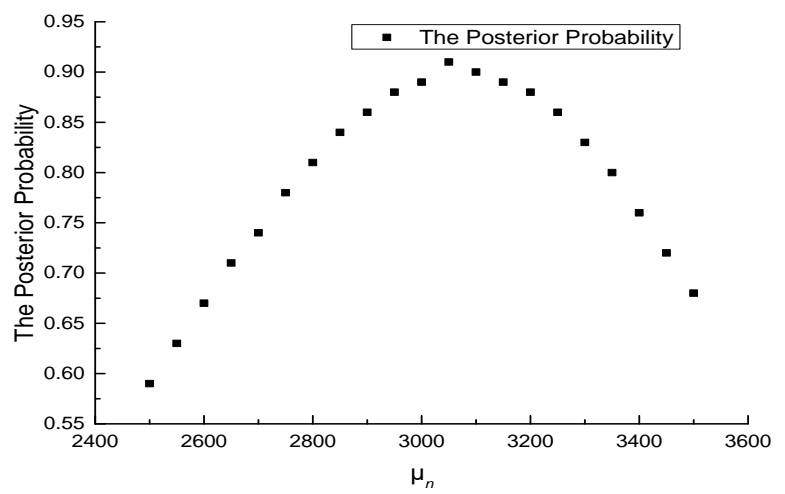

Figure 1. The posterior probability with different $\mu_{\eta}$

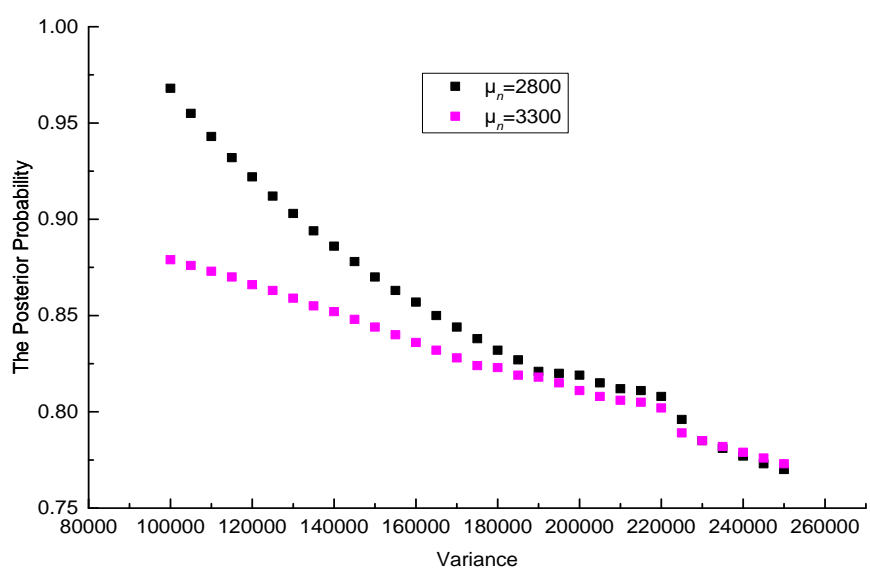

Figure 2. The posterior probability with different variances when $\mu_{\eta}=2800$ and $\mu_{\eta}=3300$ 
It can be shown that in the confidence interval $C_{\eta}=(2500,3500)$, when $\mu_{\eta}$ is changed between 2800 and 3350, it can be sure that the posterior expected loss $\rho$ is less than 0.2 .

The posterior probability of $\sigma_{\eta}^{2}$ changed from $1.0 \times 10^{5}$ to $2.5 \times 10^{5}$ are showm in Figure 2 when $\mu_{\eta}$ is 2800 and 3300 respectively. It can be known that the posterior expected loss is less than 0.2 when $\mu_{\eta}$ is changed between 2800 and 3300, $C_{\eta}=(2500,3500)$, and $\sigma_{\eta}^{2} \leq 2.2 \times 10^{5}$.

The same method can also be used to calculate the range of $\mu_{m}$ when $C_{m}=(1,1.7)$ and $\rho \leq 0.2$. Then it can be got that the value range of $\mu_{m}$ is changed between 1.1 and 1.54 .

Therefore, the prior distribution can be choosed by:

$$
\Gamma=\left\{\begin{array}{l}
\pi(m, \eta)=\frac{1}{2 \pi \sigma_{m} \sigma_{\eta}} \exp \left(-\frac{\left(m-\mu_{m}\right)^{2}}{2 \sigma_{m}^{2}}-\frac{\left(\eta-\mu_{\eta}\right)^{2}}{2 \sigma_{\eta}^{2}}\right) \\
1.1 \leq \mu_{m} \leq 1.54,2800 \leq \mu_{\eta} \leq 3300, \sigma_{m}^{2} \leq 0.05, \sigma_{\eta}^{2} \leq 2.2 \times 10^{5}
\end{array}\right\}
$$

It can be sure the posterior expected loss is less than 0.2 within the given confidence intervals if the parameters of prior distribution are taken values in a wider range. Therefore, the prior distribution is robust.

\section{Conclusion}

According to the maximum entropy principle, it takes kinds of priori information as different constraints. The optimal prior distribution is selected by maximizing entropy under these constraints. The obtained prior distribution not only contains the known prior information but also avoids the introduction of other assumption information. Taking the historical failure data of low-voltage switchgear as priori information, these hyper-parameters of prior distribution were estimated to get the prior density function by two-order moment of prior information. According to the field test data, it can be obtained that the posterior mean time between failures for the low-voltage switchgear is $3562 \mathrm{~h}$ by analyzing the reliability of low-voltage switchgear. When given the confidence intervals $C_{m}=(1,1.7), C_{n}=(2500,3500)$ and the acceptable maximum posterior expected loss $\gamma=0.2$ by experts experience, the prior distribution robustness and the posterior robustness were analyzed with the bootstrap method.

\section{Acknowledgements}

This work supported by the National Natural Science Foundation of China. No. 51077039 and Natural Science Foundation Project of Hebei Province No. E2012202111.

\section{References}

[1] Zhang SF, Fan SJ, Wang HP. Bayesian Reliability Assessment for Complex Systems. Aerospace Control. 2000; (2): 72-79.

[2] Tama BA, Rodiyatul FS, Hermansyah. An Early Detection Method of Type-2 Diabetes Mellitus in Public Hospital. Telkomnika (Telecommunication Computing Electronics and Control). 2011; 9(2): 287-294.

[3] Gopalakrishnan T, Sengottuvelan P. A Hybrid PSO With Naive Bayes Classifier for Disengagement Detection in Online Learning. Program-Electronic Library and Information Systems. 2016; 50(2): 215224.

[4] Pan P. A Bayes Approach to Reliability Prediction Utilizing Data from Accelerated Life Tests and Field Failure Observations. Quality and Reliability Engineering International. 2009; 25(2): 229-240.

[5] Novak MG, Veluscek D. Prediction of stock price movement based on daily high prices. Quantitative Finance. 2016; 16(5): 793-826.

[6] Sanchez-Cortina I, Andres-Ferrer J, Sanchis A, Juan A. Speaker-adapted confidence measures for speech recognition of video lectures. Computer Speech and Language. 2016; 37: 11-23.

[7] Chen Y, Xiong J, Liu HL, Fan, Q. Combine target extraction and enhancement methods to fuse infrared and LLL images. Telkomnika (Telecommunication Computing Electronics and Control). 2014; 12(3): 605-612.

[8] Giffin A, Cafaro C, Ali SA. Application of the Maximum Relative Entropy Method to the Physics of Ferromagnetic Materials. Physica A-Statistical Mechanics and Its Applications. 2016; 455: 11-26. 
[9] Lei M, Fan Y. Image fuzzy enhancement based on self-adaptive bee colony algorithm. Telkomnika (Telecommunication Computing Electronics and Control). 2014; 12(4): 875-882.

[10] Al-Saleh MF, Samawi HM. Estimation of the Correlation Coefficient Using Bivariate Ranked Set Sampling With Application to the Bivariate Normal Distribution. Communications in Statistics-Theory and Methods. 2005; 34(4): 875-889.

[11] Karacan CO, Goodman GVR. Probabilistic Modeling Using Bivariate Normal Distributions for Identification of Flow And Displacement Intervals in Longwall Overburden. International Journal of Rock Mechanics and Mining Sciences. 2011; 48(1): 27-41.

[12] Nicolin DJ, Rossoni DF, Jorg LMM. Study of Uncertainty in the Fitting of Diffusivity of Fick's Second Law of Diffusion with the Use of Bootstrap Method. Journal of Food Engineering. 2016; 184: 63-68.

[13] Dudek AE, Paparoditis E, Politis DN. Generalized Seasonal Tapered Block Bootstrap. Statistics \& Probability Letters. 2016; 115: 27-35.

[14] Barber JA, Thompson SG. Analysis of Cost Data in Randomized Trials: an Application of the NonParametric Bootstrap. Statistics In Medicine. 2000; 19(23): 3219-3236.

[15] Liao JG, McMurry T, Berg A. Prior Robust Empirical Bayes Inference for Large-Scale Data by Conditioning on Rank With Application to Microarray Data. Biostatistics. 2014; 15(1): 60-73.

[16] Beck JL, Taflanidis AA. Prior and Posterior Robust Stochastic Predictions for Dynamical Systems Using Probability Logic. International Journal for Uncertainty Quantification. 2013; 3(4): 271-288.

[17] Jia X, Wang D, Jiang P, Guo B. Inference on the Reliability of Weibull Distribution With Multiply TypeI Censored Data. Reliability Engineering \& System Safety. 2016; 150: 171-181. 